Mathematical Research Letters 1, 211-223 (1994)

\title{
GLOBAL AND UNIQUE WEAK SOLUTIONS OF NONLINEAR WAVE EQUATIONS
}

\section{LEV KAPITANSKI}

While the uniqueness of the energy class solutions of semi-linear wave equations with supercritical nonlinearity remains an open problem, there has been a considerable progress recently in understanding of the critical and subcritical cases (see [Brenner \& von Wahl], [Pecher], [Ginibre \& Velo 1-3], [Ginibre, Soffer \& Velo], [Kapitanski 1, 4-6], [Struwe 1, 2], [Grillakis 1-3], [Shatah \& Struwe]). In particular, the following result is proved in [Kapitanski 4, 5].

Let $\mathcal{L}$ be a second order hyperbolic differential operator of the form (the sum over the repeated indices is assumed)

$$
\begin{aligned}
\mathcal{L}=\frac{\partial^{2}}{\partial t^{2}}+a^{j, 0}(t, x) \frac{\partial^{2}}{\partial t \partial x_{j}}-a^{j, k} & (t, x) \frac{\partial^{2}}{\partial x_{j} \partial x_{k}} \\
& +a^{0}(t, x) \frac{\partial}{\partial t}+a^{j}(t, x) \frac{\partial}{\partial x_{j}}+a(t, x)
\end{aligned}
$$

where $a^{j, 0}, a^{j, k}, a^{0}, a^{j}$ and $a$ are smooth functions of $t$ and $x \in \mathbb{R}^{n}$ which do not depend on $x$ when $|x| \geq R_{0}$, for some $R_{0}>0$; the coefficients $a^{j, 0}, a^{j, k}$ are real, the matrix $\left\{a^{j, k}\right\}$ is symmetric and (uniformly with respect to $t$ and $x$ ) positive definite. Throughout the paper we assume that the number of space dimensions is $n \geq 3$.

Let $\tilde{f}: \mathbb{C} \rightarrow \mathbb{C}$ be a continuous function which can be represented as a difference,

$$
\tilde{f}(z)=f(z)-h(z)
$$

where $f$ and $h$ satisfy the following conditions. The function $f$ has an anti-derivative $F$, in the sense that

$$
f(z)=\frac{\partial F(z)}{\partial \bar{z}}
$$

Received November 5, 1993. 
where $F$ is a non-negative, real valued, continuously differentiable with respect to $z$ and $\bar{z}$ function, and

$$
\left|f\left(z_{1}\right)-f\left(z_{2}\right)\right| \leq \varkappa\left(\left|z_{1}\right|^{\sigma}+\left|z_{2}\right|^{\sigma}\right)\left|z_{1}-z_{2}\right|, \quad \forall z_{1}, z_{2} \in \mathbb{C}
$$

for some constants $\varkappa>0$ and $\sigma, 0 \leq \sigma \leq 4 /(n-2)$, while $h$ is a globally Lipschitz-continuous function, i.e.

$$
\left|h\left(z_{1}\right)-h\left(z_{2}\right)\right| \leq \varkappa\left|z_{1}-z_{2}\right|, \quad \forall z_{1}, z_{2} \in \mathbb{C} .
$$

For several reasons the exponent

$$
\sigma_{*}=\frac{4}{n-2}
$$

is called critical.

Now, consider the problem

$$
\begin{aligned}
\mathcal{L} u(t, x) & +\tilde{f}(u(t, x))=0, \\
u(0, x) & =\phi(x), \quad \dot{u}(0, x)=\psi(x) .
\end{aligned}
$$

Under the above assumptions we have the following existence and uniqueness result (see [Kapitanski 5: Theorem 0.5, and 4: Theorem 0.7]).

\section{Theorem 1.}

(1) When $0 \leq \sigma<\frac{4}{n-2}$ (the subcritical case), for any initial data $\phi \in H^{1}\left(\mathbb{R}^{n}\right)$ and $\psi \in L_{2}\left(\mathbb{R}^{n}\right)$ there exists a unique solution $u(t, x)$ of (2) with the following properties:

(i) $u$ is a strongly continuous $H^{1}$-valued function of $t$ and $\dot{u}$ is a strongly continuous $L_{2}$-valued function of $t$;

(ii) $u \in L_{\frac{2(n+1)}{n-2}}\left([0, T] \times \mathbb{R}^{n}\right)$ for every $T>0$.

(2) When $\sigma=\frac{4^{n-2}}{n-2}$ (the critical case), for any initial data $\phi \in H^{1}\left(\mathbb{R}^{n}\right)$ and $\psi \in L_{2}\left(\mathbb{R}^{n}\right)$ there exists a unique solution $u(t, x)$ of $(2)$ with the following properties:

$(\dagger) u$ is a weakly continuous $H^{1}$-valued function of $t$ and $\dot{u}$ is a weakly continuous $L_{2}$-valued function of $t$; but for every $t \geq 0$ there exists a $\tau(t)>0$ such that

(i) $u$ is a strongly continuous $H^{1}$-valued function of $t$ on the interval $[t, t+\tau(t)]$ and $\dot{u}$ is a strongly continuous $L_{2}$-valued function of $t$ on $[t, t+\tau(t)]$;

(ii) $u \in L_{\frac{2(n+1)}{n-2}}\left([t, t+\tau(t)] \times \mathbb{R}^{n}\right)$. 
To the best of the author's knowledge, in the critical case, $\sigma=4 /(n-2)$, except for Theorem 1 there are no other unconditional results on the global and unique weak solutions even for the nonlinear Klein-Gordon equation (i.e. (2a) with $\mathcal{L}=\square=$ the standard d'Alembertian). However, the second (critical case) statement of Theorem 1 does not look natural at all. For example, it does not exclude a possibility for a weak solution from the uniqueness class described in Theorem 1 to have the "energy"

$$
E[u](t)=\frac{1}{2} \int d x\left(|\dot{u}(t, x)|^{2}+\left|\nabla_{x} u(t, x)\right|^{2}+|u(t, x)|^{2}+F(u(t, x))\right)
$$

with a countable number of discontinuities in $t$ (the discontinuities are allowed even to have accumulating points!). It is not clear so far whether the solutions with discontinuous energy exist for the general problem (2). However, there have been some indications that for the problem

$$
\begin{gathered}
\square u(t, x)+u(t, x)+\tilde{f}(u(t, x))=0, \\
u(0, x)=\phi(x), \quad \dot{u}(0, x)=\psi(x),
\end{gathered}
$$

the situation is better. Namely, it is shown in [Ginibre, Soffer \& Velo, Proposition 4.3] that in the case of spherically-symmetric weak solutions of (4) with

$$
\tilde{f}(u)=f(u)=|u|^{\sigma_{*}} u,
$$

the energy remains constant (hence, continuous) for all $t>0$. On the other hand, in dimensions $3 \leq n \leq 7$ the results of [Struwe 1, 2], [Grillakis 1-3] and [Shatah \& Struwe] give the existence of global regular $\left(=C^{\infty}\right)$ solutions of $(4)+(5)$ for all regular initial data.

The main goal of the present paper is to show for problem (4) with a critical nonlinearity $\tilde{f}$ that under certain additional assumptions on $\tilde{f}$, all the weak solutions with the properties described in the second part of Theorem 1 have a continuous in $t$ energy $E[u](t)$. Before we proceed with the exact statements on this matter, let us discuss the whole situation briefly. From now on we will always assume that $f$ enjoys (1c) with the critical exponent $\sigma=\sigma_{*}=4 /(n-2)$.

Consider the general problem (2). Let $\mathcal{A}$ denote the class of solutions of problem (2) (corresponding to all possible initial data $\phi \in H^{1}, \psi \in L_{2}$ ) with the properties $(\dagger)$, (i) and (ii) of the second part of Theorem 1. For $u$ in $\mathcal{A}$ denote by $\mathcal{T}[u]$ the (at most countable) set of all $\tau>0$ where $E[u](\tau-0) \neq E[u](\tau+0)$. We have the following result. 
Proposition 2. Given $u \in \mathcal{A}$ and $t_{0} \geq 0$, let $T>t_{0}$ be such that the open interval $\left(t_{0}, T\right)$ contains no points of $\mathcal{T}[u]$.

The following properties of $u$ are equivalent.

(i) $T \notin \mathcal{T}[u]$;

(ii) $\{u, \dot{u}\} \in C\left(\left[t_{0}, T\right] \rightarrow H^{1} \times L_{2}\right)$;

(iii) $u \in L_{\frac{2(n+1)}{n}}\left(\left[t_{0}, T\right] \times \mathbb{R}^{n}\right)$;

(iv) $u \in L_{q_{*}}{ }^{n-2}\left(\left[t_{0}, T\right] \rightarrow L_{p_{*}}\left(\mathbb{R}^{n}\right)\right)$, for all $p_{*}$ and $q_{*}$ such that

$$
\frac{2 n}{n-2}<p_{*}<\frac{2 n}{n-3}, \quad \frac{1}{q_{*}}=n\left(\frac{1}{2}-\frac{1}{p_{*}}\right)-1 ;
$$

(v) $u \in L_{q}\left(\left[t_{0}, T\right] \rightarrow B_{p}^{r}\left(\mathbb{R}^{n}\right)\right)$, for all $r, p$ and $q$ such that

$$
1-\frac{n+1}{2(n-1)}<r<1, \quad \frac{1}{p}=\frac{1}{2}-\frac{2}{n+1}(1-r), \quad \frac{1}{q}=\frac{n-1}{n+1}(1-r) ;
$$

here $B_{p}^{r}$ is the Besov space $B_{p, 2}^{r}$, see [Triebel] for definition and basic properties of these spaces;

(vi) For every $x^{0} \in \mathbb{R}^{n}$ there exist $\delta=\delta\left(u ; x^{0}\right)>0$ and a ball $\Omega\left(x^{0}\right)=$ $\left\{x:\left|x-x^{0}\right| \leq \rho\right\}, \rho=\rho\left(u ; x^{0}\right)>0$, such that

$$
u \in L_{\frac{2(n+1)}{n-2}}\left([T-\delta, T] \times \Omega\left(x^{0}\right)\right) ;
$$

(vii) For every $x^{0} \in \mathbb{R}^{n}$ there exists $\delta=\delta\left(u ; x^{0}\right)>0$ such that

$$
\int_{T-\delta}^{T} d t \int_{\delta\left|x-x^{0}\right|^{2} \leq T-t}|u(t, x)|^{\frac{2(n+1)}{n-2}} d x<\infty,
$$

while the hypersurface $\Sigma_{\delta}=\left\{t=T-\delta|x|^{2}\right\}$ is space-like with respect to $a^{\mu \nu}(t, x)$ in the sense that

(8) $\quad\left(\nu_{0}(t, x)+\nu_{j}(t, x) a^{j, 0}(t, x)\right) \xi_{0}^{2}-2 \nu_{j}(t, x) a^{j, k}(t, x) \xi_{k} \xi_{0}$

$$
+\nu_{0}(t, x) a^{j, k}(t, x) \xi_{j} \xi_{k}>0
$$

for all $\xi \in \mathbb{R}^{1+n}, \xi \neq 0$, and all $(t, x)$ on the hypersurface, where $\nu(t, x)$ is the unit outer (means $\nu_{0}(t, x)>0$ ) normal to $\Sigma_{\delta}$ at $(t, x)$.

Proof. By the definition of the set $\mathcal{T}[u]$ we have (i) $\Leftrightarrow($ ii). That $(\mathrm{v}) \Leftrightarrow($ ii) follows from [Kapitanski 5, subsections 3.4 and 3.6]. Now, (v) $\Rightarrow$ (iv) by 
embedding theorems, and (iii) is a particular case of (iv). Let us show that (iii) $\Rightarrow$ (ii). Indeed, by assumption $u \in \mathcal{A}$ and there are no points of $\mathcal{T}[u]$ in $\left(t_{0}, T\right)$. Therefore, $\{u, \dot{u}\} \in C\left(\left[t_{0}, t\right] \rightarrow H^{1} \times L_{2}\right)$, for all $t<T$. Besides, as $t \nearrow T$ there is a unique weak limit of $\{u(t), \dot{u}(t)\}$ in $H^{1} \times L_{2}$. Let us take this limit $\{u(T), \dot{u}(T)\}$ as new initial data and solve equation (2a) backward in time. We thus will obtain, by Theorem 1, a unique solution, say, $\underline{u}$, such that, first, $\{\underline{u}, \underline{\dot{u}}\} \in C\left([\tau, T] \rightarrow H^{1} \times L_{2}\right)$ and, second, $\underline{u} \in$ $L_{\frac{2(n+1)}{n-2}}\left([\tau, T] \times \mathbb{R}^{n}\right)$, for some $\tau<T$ (we may assume that $\left.\tau \geq t_{0}\right)$. Since both $u$ and $\underline{u}$ are weak solutions to $(2 \mathrm{a})$ on $[\tau, T]$ with common initial data

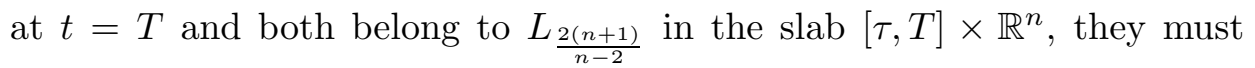
coincide, by (uniqueness) Theorem 2.1 of [Kapitanski 4].

Now let us show that (vi) $\Rightarrow($ ii). First note that from the original hypotheses on $u$ and the interval $\left(t_{0}, T\right)$, assumptions (vi) imply that $u \in$ $L_{\frac{2(n+1)}{n-2}}\left(\left[t_{0}, T\right] \times\{|x| \leq R\}\right)$ for an arbitrary $R>0$. The rest of the proof is similar to that of (iii) $\Rightarrow$ (ii) except that instead of the global uniqueness theorem one has to use its local version which we state below for convenience of the reader.

Theorem 3. Let operator $\mathcal{L}$ satisfy the same conditions as above and function $\tilde{f}$ enjoys the properties (1) with $\sigma=\sigma_{*}$. Let $u_{j}, j=1,2$, be two weak solutions of equation (2a) in the slab $\Pi=[0, T] \times \mathbb{R}^{n}$ (this means, in particular, that $\left.\left\{u_{j}, \dot{u}_{j}\right\} \in L_{\infty}\left([0, T] \rightarrow H^{1} \times L_{2}\right)\right)$, with some initial conditions $\phi_{j} \in H^{1}, \psi_{j} \in L_{2}$. Assume that $\phi_{1}=\phi_{2}$ and $\psi_{1}=\psi_{2}$ in a domain $Q_{0} \subset \mathbb{R}^{n}$, and it is known a priori that both $u_{1}$ and $u_{2}$ belong to $L_{\frac{2(n+1)}{n-2}}$ in a space-time region $Q=\left\{(t, x): 0 \leq t \leq T, x \in Q_{t}\right\}$, whose lateral boundary $\partial_{l} Q=\{(t, x) \in \partial Q, 0<t<T\}=\left\{(t, x): 0<t<T, x \in \partial Q_{t}\right\}$, is space or null oriented with respect to the metric $a^{\mu \nu}(t, x)$ (i.e. for every $(t, x) \in \partial_{l} Q$, either a strict inequality (8) or the corresponding equality takes place).

It follows then that $u_{1}=u_{2}$ in $Q$.

Proof. The proof goes along the same lines as that of Theorem 2.1 of [Kapitanski 4], but the treatment of the almost-linear $h(u)$-term requires a slight modification, and while working with $f(u)$ one should replace the "standard" Strichartz estimate (see [Kapitanski 4, Theorem 1.2]) by its localized in space-time version, namely,

$$
\left(\int_{\tau}^{\tau+\epsilon} d t \int_{x \in Q_{t}}|w(t, x)|^{\frac{2(n+1)}{n-1}} d x\right)^{\frac{n-1}{2(n+1)}} \leq C\left(\int_{\tau}^{\tau+\epsilon} d t \int_{x \in Q_{t}}|g(t, x)|^{\frac{2(n+1)}{n+3}} d x\right)^{\frac{n+3}{2(n+1)}},
$$


for a solution $w$ of the linear problem $\mathcal{L} w=g$ with zero initial conditions at $t=\tau$; the constants $\epsilon$ and $C$ are independent of the choice of $\tau \in[0, T)$ and $g \in L_{\frac{2(n+1)}{n+3}}(Q)$.

Proof of Proposition 2 (continued). In order to prove the implication $(\mathrm{vi}) \Rightarrow(\mathrm{ii})$, take again a unique backward in time solution $\underline{u}$ of $(2 \mathrm{a})$ which assumes the same (initial) values at $t=T$ as $u$, and has the properties, first, $\{\underline{u}, \underline{\dot{u}}\} \in C\left([\tau, T] \rightarrow H^{1} \times L_{2}\right)$ and, second, $\underline{u} \in L_{\frac{2(n+1)}{n-2}}\left([\tau, T] \times \mathbb{R}^{n}\right)$, for some $\tau<T$. Observe now that by Theorem 3, $\underline{u}=u$ in the intersection of $\Pi=[\tau, T] \times \mathbb{R}^{n}$ with a cone $K=\{(t, x):|x| \leq R-V(T-t)\}$ (its tip pointed downward), where $R$ is arbitrarily large and $V>0$ is such that

$$
\begin{aligned}
\left(V-\frac{x_{j}}{|x|} a^{j, 0}(t, x)\right) \xi_{0}^{2}+2 \frac{x_{j}}{|x|} a^{j, k}(t, x) \xi_{k} \xi_{0} & \\
& +V a^{j, k}(t, x) \xi_{j} \xi_{k} \geq 0, \quad \forall \xi \in \mathbb{R}^{1+n}
\end{aligned}
$$

for all $(t, x) \in \Pi$ (this is possible because of the assumptions on $\mathcal{L}$ ).

It remains to prove that (vii) implies (vi). Since the arguments are local, we shall fix an arbitrary $x^{0}$, say, $x^{0}=0$, and show that $u \in L_{\frac{2(n+1)}{n-2}}([T-$ $\rho, T] \times\{|x| \leq \rho\})$ for some $\rho>0$.

Denote $Q=Q^{\delta}=\left\{(t, x): x \in \mathbb{R}^{n}, 0 \leq t \leq \Phi^{\delta}(x)\right\}$, where $\Phi^{\delta}$ is a $C^{\infty}$ function with the following properties: it is radial, non-increasing, $\Phi^{\delta}(x)=T-\delta|x|^{2}$, for $|x| \leq 1$, and $\Phi^{\delta}(x)=T-2 \delta$, for $|x| \geq M$, where $\delta>0$ is small enough while $M>0$ is sufficiently large so that $\Sigma=\left\{t=\Phi^{\delta}(x)\right\}$ is space-like.

It is clear that there exists a smooth diffeomorphism (change of variables) $\Psi:(t, x) \rightarrow\left(t^{\prime}, x^{\prime}\right)$, of a slab $[-1, T+1] \times \mathbb{R}^{n}$ onto its image, such that $Q^{\prime}=$ $\Psi(Q)=[0, T-2 \delta] \times \mathbb{R}^{n}, \Sigma^{\prime}=\Psi(\Sigma)=\left\{t^{\prime}=T-2 \delta\right\}$, and $\left(t^{\prime}, x^{\prime}\right)=(t, x)$, for $|x| \geq 10 \mathrm{M}$. Under this diffeomorphism, the solution $u$ of problem (2) in $Q$ transforms into a solution $\tilde{u}$ of a similar problem in the slab $Q^{\prime}$. By assumption, $\tilde{u} \in L_{\frac{2(n+1)}{n-2}}\left(Q^{\prime}\right)$. Hence, by the already proved part of Proposition 2, $\tilde{u}$ can be extended to a solution in a larger slab, say, $Q^{\prime \prime}=[0, T-2 \delta+\epsilon] \times \mathbb{R}^{n}$. Therefore, the inverse change of variables $\Psi^{-1}$ will transform $\tilde{u}$ into an extension of $u$ to a larger domain which contains

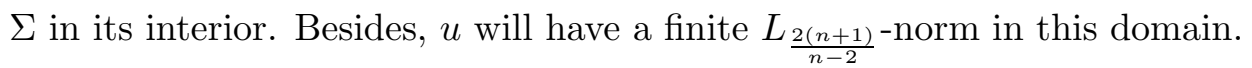
The proof is completed.

Remark. Under the same assumptions $\left(u \in \mathcal{A}\right.$ and $\left.\left(t_{0}, T\right) \cap \mathcal{T}[u]=\varnothing\right)$ it is possible to show that the following property is also equivalent to any 
of the properties (i)-(vii) of Proposition 2: for every $x^{0} \in \mathbb{R}^{n}$ there exist $\delta=\delta\left(u ; x^{0}\right)>0$ and $\Omega=\Omega\left(u ; x^{0}\right)=\left\{x:\left|x-x^{0}\right| \leq \rho\right\}, \rho=\rho\left(u ; x^{0}\right)>0$, such that $u \in C\left([T-\delta, T] \rightarrow H^{1}(\Omega)\right)$ and $\dot{u} \in C\left([T-\delta, T] \rightarrow L_{2}(\Omega)\right)$.

The proof uses certain localized Strichartz estimates (see below) and follows the same lines as the arguments of subsection 3.6 of [Kapitanski 5]. The reason I have mentioned this fact is to support the statement that for the solutions $u \in \mathcal{A}$ the continuity of the global energy $E[u](t)$ is equivalent to that of the local energy $E_{\Omega}[u](t)$,

$$
E_{\Omega}[u](t)=\frac{1}{2} \int_{\Omega} d x\left(|\dot{u}(t, x)|^{2}+\left|\nabla_{x} u(t, x)\right|^{2}+|u(t, x)|^{2}+F(u(t, x))\right),
$$

for all balls $\Omega$ in $\mathbb{R}^{n}$. Given $\Omega$, we know that $E_{\Omega}[u](t)$ is bounded uniformly with respect to $t \in\left[t_{0}, T\right)$. Thus, for $E_{\Omega}[u](t)$ to have a limit $E_{\Omega}[u](T)$ as $t \nearrow T$ is equivalent to the uniform integrability of the energy densities $e[u](t, \cdot)=\frac{1}{2}\left(|\dot{u}(t, \cdot)|^{2}+\left|\nabla_{x} u(t, \cdot)\right|^{2}+|u(t, \cdot)|^{2}+F(u(t, \cdot))\right)$ in $\Omega$, or, in other words, to the non-concentration of energy, which means that for every $\epsilon>0$ there exists $\delta>0$ such that $E_{\omega}[u](t) \leq \epsilon$ for all $t \in\left[t_{0}, T\right)$ provided the measure of $\omega \subset \Omega$ is less than $\delta$.

I already knew all these facts at the time when paper [Kapitanski 5] was in preparation. What I didn't know was how to justify the nonconcentration of energy. Meanwhile, in the pioneering paper [Struwe 1] followed by the works [Grillakis 1, 3], dealing with the regularity problem for the nonlinear Klein-Gordon equation (4a), a similar difficulty has been successfully overcome. It was M. Struwe who pointed out the significance of the local (in space-time) considerations in the study of the critically nonlinear wave equations and showed once again the importance of the local Morawetz estimates. M. Grillakis on his part made a breakthrough observation that, when combined with the Strichartz estimates, the non-concentration of the $L_{\frac{2 n}{n-2}}$-norm of a solution alone is sufficient for regularity. He showed this in the case $3 \leq n \leq 5$, see [Grillakis 3]. Shortly after, J. Shatah and M. Struwe used a different Strichartz estimate to settle the regularity in the case $n=6,7$, see [Shatah \& Struwe]. In what follows I will try to convince the reader that the approach outlined in [Grillakis 3] works for the weak solutions as well (see Theorem 7 below). It also works for the $C^{\infty}$ solutions in dimensions up to $n=9$ if one invokes the more elaborate (than in [Grillakis 3] and [Shatah \& Struwe]) Strichartz estimates (see Theorem 8 below). We shall be clinging to the general problem (2) as long as possible. 
Given $T>0$, let $Q$ be as in the final part of the proof of Proposition 2 . The notation $Q_{\tau}$ will be used for a cross-section $Q \cap\{t=\tau\}$.

Proposition 4. Let $u \in \mathcal{A}$ be given and let $T>0$ be such that $(0, T) \cap$ $\mathcal{T}[u]=\varnothing$. Assume that

$$
\int_{Q_{t}}|u(t, x)|^{\frac{2 n}{n-2}} d x \underset{t \nearrow T}{\longrightarrow} 0
$$

Then $u \in L_{\frac{2(n+1)}{n-2}}(Q)$.

Proof. We shall need certain local Strichartz estimates of the following type.

\section{Lemma 5.}

1) For every $p_{*}$ and $q_{*}$ obeying (6) there is a constant $C>0$ such that

$$
\begin{aligned}
& \left.\int_{\tau}^{T}\|v(t)\|_{L_{p_{*}}\left(Q_{t}\right)}^{q_{*}} d t\right)^{\frac{1}{q_{*}}} \\
& \quad \leq C\left\{\|v(\tau)\|_{H^{1}\left(Q_{\tau}\right)}+\|\dot{v}(\tau)\|_{L_{2}\left(Q_{\tau}\right)}+\int_{\tau}^{T}\|\mathcal{L} v(t)\|_{L_{2}\left(Q_{t}\right)} d t\right\},
\end{aligned}
$$

for all $\tau \in[T-\delta, T]$ and for all smooth $v$.

2) Let $r, p, q, \bar{r}, p^{\prime}$ and $q^{\prime}$ be as follows,

$$
\begin{gathered}
p=2^{*}=\frac{2 n}{n-2}, \quad r=\frac{1}{q}=\frac{n-1}{2 n}, \quad p^{\prime}=\frac{2 n}{n+2}, \\
\bar{r}=1-r=\frac{1}{q^{\prime}}=\frac{n+1}{2 n} .
\end{gathered}
$$

In the case $n \geq 5$, there exist $\epsilon>0$ and $C>0$ such that

$$
\begin{aligned}
& \left(\int_{\tau}^{\tau+\theta}\|v(t, \cdot)\|_{B_{p}^{r}\left(Q_{t}\right)}^{q} d t\right)^{\frac{1}{q}} \leq C\left\{\|v(\tau, \cdot)\|_{H^{1}\left(Q_{\tau}\right)}+\|\dot{v}(\tau)\|_{L_{2}\left(Q_{\tau}\right)}\right. \\
& \left.+\int_{\tau}^{\tau+\theta}\|\mathcal{L} v(t, \cdot)\|_{L_{2}\left(Q_{t}\right)} d t \wedge\left(\int_{\tau}^{\tau+\theta}\|\mathcal{L} v(t, \cdot)\|_{B_{p^{\prime}}^{\bar{r}}\left(Q_{t}\right)}^{q^{\prime}} d t\right)^{\frac{1}{q^{\prime}}}\right\}
\end{aligned}
$$

for all $\theta \leq \epsilon_{0}$, for all $\tau \in[T-\delta, T-\theta]$, and for all smooth $v$; here notation $B_{p}^{r}\left(Q_{t}\right)$ is used for a local Besov space $B_{p, 2}^{r}\left(Q_{t}\right)$ in the ball $Q_{t}$ ( see [Triebel, Section 3]), and $a \wedge b$ stands for the minimum of $a$ and $b$.

Proof. Lemma follows from the corresponding global results (see [Kapitanski 5, Section 1], and [Kapitanski 3] for more general results), and the finite 
domain of dependence property of hyperbolic differential equations (Theorem 3, linear case). For the second assertion one needs in addition to show that there exist extension operators $\mathcal{E}_{t}: B_{p}^{r}\left(Q_{t}\right) \rightarrow B_{p}^{r}\left(\mathbb{R}^{n}\right)$ which are, together with the restriction operators $\mathcal{R}_{t}: B_{p}^{r}\left(\mathbb{R}^{n}\right) \rightarrow B_{p}^{r}\left(Q_{t}\right)$, uniformly in $t$ bounded. It is not hard to construct the required $\mathcal{E}_{t}$ in the case when $Q_{t}$ 's are balls and $0<r<1,1<p<n$. I do not know whether such extensions exist in more general situations. This explains why the second assertion is so restrictive.

Proof of Proposition 4, continued. In the case $n=3$, 4, or 5, the proof uses estimate (10) and is essentially the same as in [Grillakis 3]. So we shall consider the case $n \geq 6$. Note that it is sufficient to prove that

$$
\int_{T-\delta}^{T}\|u(t, \cdot)\|_{B_{p}^{r}\left(Q_{t}\right)}^{q} d t<\infty,
$$

where $r, p$, and $q$ are defined in (11). Indeed, since $B_{p}^{r} \subset L_{p_{*}}$, where $p_{*}=$ $\frac{2 n^{2}}{n^{2}-3 n+1}$, the estimate (13) will imply that $u \in L_{q}\left(t \rightarrow L_{p_{*}}\left(Q_{t}\right)\right)$. At the same time $u \in L_{\infty}\left(t \rightarrow L_{2^{*}}\left(Q_{t}\right)\right)$, and we get $u \in L_{\frac{2(n+1)}{n-2}}\left(t \rightarrow L_{\frac{2(n+1)}{n-2}}\left(Q_{t}\right)\right)$ via interpolation.

In order to prove (13) we will show that for any $\theta>0$, the norms

$$
N_{\tau}:=\left(\int_{\tau}^{(\tau+\theta) \wedge T}\|u(t, \cdot)\|_{B_{p}^{r}\left(Q_{t}\right)}^{q} d t\right)^{\frac{1}{q}}
$$

are uniformly bounded when $\tau$ is close to $T$. Indeed, applying (12) to $u$ we obtain

$$
\begin{aligned}
N_{\tau} \leq & C\left\{\|u(\tau)\|_{H^{1}\left(Q_{\tau}\right)}+\|\dot{u}(\tau)\|_{L_{2}\left(Q_{\tau}\right)}\right. \\
& \left.+\int_{\tau}^{\tau+\theta}\|h(u(t))\|_{L_{2}\left(Q_{t}\right)} d t+\left(\int_{\tau}^{\tau+\theta}\|f(u(t))\|_{B_{p^{\prime}}^{\bar{r}^{\prime}}\left(Q_{t}\right)}^{q^{\prime}} d t\right)^{\frac{1}{q^{\prime}}}\right\} .
\end{aligned}
$$

Each of the first three terms on the right is bounded by the supremum of the energy $E[u](t)$ on $[0, T]$. Using Lemma 3.2 of [Ginibre \& Velo 1], certain embedding theorems and interpolation (the arguments are similar to those employed in the proof of Lemma 2.8 in [Kapitanski 5], but much simpler), one can estimate the last term on the right as follows:

$$
\begin{aligned}
& \left(\int_{\tau}^{\tau+\theta}\|f(u(t))\|_{B_{p^{\prime}}^{\bar{r}}\left(Q_{t}\right)}^{q^{\prime}} d t\right)^{\frac{1}{q^{\prime}}} \\
& \quad \leq c \sup _{\tau \leq t \leq \tau+\theta}\|u(t)\|_{L_{2^{*}}\left(Q_{t}\right)}^{\sigma_{*}(1-\lambda)} \cdot \sup _{\tau \leq t \leq \tau+\theta}\|u(t)\|_{H^{1}\left(Q_{t}\right)}^{1-\nu} \cdot\left(N_{\tau}\right)^{\gamma},
\end{aligned}
$$


where

$$
\lambda=\frac{n(n-2)}{n^{2}-1}, \quad \nu=\frac{n-1}{n+1}, \quad \gamma=\nu+\sigma_{*} \lambda=\frac{n+1}{n-1} .
$$

Due to our assumption (9), from (15) we finally obtain an estimate

$$
N_{\tau} \leq C_{1}+C_{2}(\tau)\left(N_{\tau}\right)^{\gamma}
$$

where $C_{1}=$ const $>0$ and $C_{2}(\tau)$ goes to 0 as $\tau$ approaches $T$. We conclude that $N_{\tau}$ is uniformly bounded. This completes the proof of Proposition 4.

Next step is to obtain (9). Unfortunately, I do not know how to do this in the general case of system (2). However, for nonlinear Klein-Gordon equation (4a) with additional assumptions on $f,(9)$ is essentially known (see [Grillakis 3, Theorem 1.5]; although Grillakis works with smooth solutions, it is not hard to modify his arguments so that to handle weak solutions as well). Thus, we have

Proposition 6. Let $u$ be a solution of (4) of class $\mathcal{A}$. Let $T>0$ be such that $(0, T) \cap \mathcal{T}[u]=\varnothing$. Assume that $f$, in addition to (1b) and (1c), satisfy the following condition: there is a constant $c>0$ such that

$$
F(z) \geq c|z|^{2^{*}}, \quad \operatorname{Re}(\bar{z} f(z))-F(z) \geq c|z|^{2^{*}}
$$

for all sufficiently large $|z|$. In this case (9) takes place for any paraboloid $Q=\left\{(t, x): \delta|x|^{2} \leq T-t, t \leq T\right\}$.

Remark. Note that (1) and (16) together imply that for large $|z|, F(z)$ is gripped between $c|z|^{2^{*}}$ and $\varkappa|z|^{2^{*}}$, for some $c, \varkappa>0$. M. Grillakis told me in May of 1991 that he had encountered a restriction of this type when working on [Grillakis 3]. It would be interesting to see whether (16) is necessary.

Summing up all the previous, we get the following result.

Theorem 7. Under the assumptions (1) and (16) on the nonlinearity $\tilde{f}$, for every $\{\phi, \psi\} \in H^{1} \times L_{2}$ there exists a unique global weak solution $u$ of problem (4) with the following properties: $u \in L_{\frac{2(n+1)}{n-2}}\left([0, T] \times \mathbb{R}^{n}\right)$ and $\{u, \dot{u}\} \in C\left([0, T] \rightarrow H^{1} \times L_{2}\right)$, for any $T>0$.

The next theorem gives certain additional properties of solutions. 
Theorem 8. Let $f$ satisfy the same conditions as in Theorem 7 .

1) The solutions of problem (4) provided by Theorem 7 depend continuously (in $C\left([0, T] \rightarrow H^{1} \times L_{2}\right)$, any $\left.T\right)$ on the initial data;

2) If $u$ is a solution of (4) provided by Theorem 7 , then $u \in L_{q_{*}}([0, T] \rightarrow$ $\left.L_{p_{*}}\left(\mathbb{R}^{n}\right)\right)$, for all $p_{*}$ and $q_{*}$ enjoying (6), and all $T>0$; besides, $u \in$ $L_{q}\left(\left[t_{0}, T\right] \rightarrow B_{p}^{r}\left(\mathbb{R}^{n}\right)\right)$, for all $r, p$ and $q$ obeying $(7) ;$

3) If the initial data $\{\phi, \psi\}$ belong to $H^{1+s} \times H^{s}$, for some $s, 0<s \leq \frac{1}{2}$, then the corresponding solution $u$ of (4) is also more regular, $\{u, \dot{u}\} \in$ $C\left([0, T] \rightarrow H^{1+s} \times H^{s}\right)$; besides, $u \in L_{q}\left(\left[t_{0}, T\right] \rightarrow B_{p}^{s+r}\left(\mathbb{R}^{n}\right)\right)$, for all $r, p$ and $q$ obeying $(7)$;

4) If $n \leq 21$, the nonlinearities $h$ and $f$ satisfy the conditions $(\mathrm{H} 4,1)$ and (F5) of [Kapitanski 5], and $\{\phi, \psi\} \in H^{2} \times H^{1}$, then the corresponding solution is strong, i.e. $\{u, \dot{u}\} \in C\left([0, T] \rightarrow H^{2} \times H^{1}\right)$;

5) If $n \leq 9$, the nonlinearity $\tilde{f}$ is infinitely differentiable and its derivatives behave at infinity like the derivatives of a power function $|z|^{\sigma_{*}} z$ should, and if $\{\phi, \psi\} \in \cap_{s>0} H^{1+s} \times H^{s}$, then $\{u, \dot{u}\} \in C\left([0, T] \rightarrow H^{1+s} \times H^{s}\right)$, for any $s>0$;

6) If $n \leq 9$, the nonlinearity $\tilde{f}$ is as in 5), and $\phi \in C^{\infty}, \psi \in C^{\infty}$, then $u \in C^{\infty}\left(\mathbb{R}^{1+n}\right)$.

Sketch of the Proof. The arguments needed to justify 1) are very similar to those used in [Kapitanski 5, Sec. 3], there one can also find the proof of 2). The assertions 3) and 4) follow from [Kapitanski 5, Theorem 0.10, see conditions (0.16), (0.17)]. Now, 5) follows from [Ginibre \& Velo 3, Proposition 3.1], while 6) is a consequence of 5).

\section{Acknowledgement}

The principal part of this paper was done when I was visiting Princeton University in the 1991-92 academic year, and I avail myself of the opportunity to thank the faculty and staff for their hospitality. My special thanks are to Sergiu Klainerman for many valuable discussions. The idea of the proof of Theorem 7 was inspired by M. Grillakis' lecture and subsequent discussion at the conference "Nonlinear Evolution Equations" in Oberwolfach, in May 1991, where he explained in detail his work [Grillakis 3], which was at that time new and unpublished. I appreciate his munificence very much indeed. During the 1992-93 academic year, when visiting Brown University, I discussed the results presented here with Walter Strauss, and I 
am grateful for his interest and moral support.

Note added in proof. After this work was finished and submitted for publication,

J. Shatah and M. Struwe informed the author that they had also obtained a result similar to our Theorem 7 .

\section{References}

P. Brenner \& W. von Wahl, Global classical solutions of nonlinear wave equations, Math. Z. 176 (1981), 87-121.

J. Ginibre, A. Soffer \& G. Velo, The global Cauchy problem for the critical non-linear wave equation, J. Funct. Anal. 110 (1992), 96-130.

J. Ginibre \& G. Velo 1, The global Cauchy problem for the nonlinear Klein-Gordon equation, Math. Z. 189 (1985), 487-505.

J. Ginibre \& G. Velo 2, The global Cauchy problem for the nonlinear Klein-Gordon equation, revised, Ann. Inst. H. Poincaré, Analyse Non Linéaire 6 (1989), 15-35.

J. Ginibre \& G. Velo 3, Regularity of solutions of critical and subcritical non linear wave equations, Preprint, LPTHE 92/06.

M. G. Grillakis 1, Regularity and asymptotic behavior of the wave equation with a critical nonlinearity, Ann. of Math. 132 (1990), 485-509.

M. G. Grillakis 2, Some remarks on the regularity of wave equations with critical nonlinearity, In: Nonlinear hyperbolic equations and field theory. Eds.: M.K.V. Murthy and S. Spagnolo (Pitman Research Notes in Mathematics Series, Vol. 253) (1992), 110-120.

M. G. Grillakis 3, Regularity for the wave equation with a critical nonlinearity, Comm. Pure Appl. Math. 45 (1992), 749-774.

L. V. Kapitanski 1, The Cauchy problem for a semilinear wave equation, I, J. Soviet Math. 49 (1990), no. 5, 1166-1186.

L. V. Kapitanski 2, Some generalizations of the Strichartz-Brenner inequality, Leningrad Math. J. 1 (1990), no. 3, 693-726.

L. V. Kapitanski 3, Norm estimates in Besov and Lizorkin-Triebel spaces for the solutions of the second-order linear hyperbolic equations, J. Soviet Math. 56 (1991), no. 2, 2348-2389.

L. V. Kapitanski 4, The Cauchy problem for a semilinear wave equation, II, J. Soviet Math. 62 (1992), no. 3, 2746-2777.

L. V. Kapitanski 5, The Cauchy problem for a semilinear wave equation, III, J. Soviet Math. 62 (1992), no. 2, 2619-2645.

L. V. Kapitanski 6, Weak and yet weaker solutions of semilinear wave equations, Preprint LCDS\# 93-3, Brown University (March 1993).

L. V. Kapitanski 7, Global weak and more regular solutions of semilinear wave equation, in preparation.

C. S. Morawetz, Time decay for the nonlinear Klein-Gordon equation, Proc. Roy. Soc. A. 306 (1968), 291-296.

H. Pecher, $L^{p}$-Abschätzungen und klassische Lösungen für nichtlineare Wellengleichungen,I, Math. Z. 150 (1976), 159-183.

J. Shatah \& M. Struwe, Regularity result for nonlinear wave equations, Preprint (1991).

W. A. Strauss, On weak solutions of semilinear hyperbolic equations, An. Acad. Brasil. Cienc. 42 (1970), no. 4, 645-651. 
R. S. Strichartz 1, Convolution with kernels having singularities on a sphere, Trans. AMS 148 (1970), 461-471.

R. S. Strichartz 2, A priori estimates for the wave equation and some applications, Journ. of Funct. Anal. 5 (1970), 218-235.

R. S. Strichartz 3, Restrictions of Fourier transforms to quadratic surfaces and decay of solutions of wave equations, Duke Math. J. 44 (1977), 705-714.

M. Struwe 1, Globally regular solutions to the $u^{5}$ Klein-Gordon equation, Ann. Sc. Norm. Sup. Pisa (Ser.4) 15 (1988), 495-513.

M. Struwe 2, Semilinear wave equations, Bull. AMS 26 (1992), 53-85.

H. Triebel, Theory of function spaces, Monographs in Mathematics, Vol. 78, Birkhäuser, Basel: Boston: Stuttgart, 1983.

Steklov Institute of M athematics, St. Peteriburg Branch, St. PetersBurg, 191011 , Rusia, and Department of Mathematics, Kansas State UniVersity, Manhattan, KS 66506 , U.S.A.

Current address: Department of Mathematics, Kansas State University, Manhattan, KS 66506, U.S.A.

E-mail address: levkapit@math.ksu.edu 